\title{
Kinematics of the Feline Antebrachiocarpal Joint from Supination to Pronation
}

\author{
Rachel M. Basa10 William C.H. Parr ${ }^{2}$ Christopher J. Tan ${ }^{1}$ William R. Walsh ${ }^{2}$ Kenneth A. Johnson ${ }^{10}$ \\ ${ }^{1}$ Sydney School of Veterinary Science, Faculty of Science, University \\ of Sydney, New South Wales, Australia \\ 2 Surgical and Orthopaedic Research Laboratories, Prince of Wales \\ Clinical School, University of New South Wales, Randwick, \\ New South Wales, Australia \\ Address for correspondence Rachel M. Basa, BVSc, DECVS, MVCS, \\ MANZCVS, Sydney School of Veterinary Science, Faculty of Science, \\ University of Sydney, New South Wales 2006, Australia \\ (e-mail: rachel.basa@sydney.edu.au). \\ Vet Comp Orthop Traumatol 2021;34:115-123.
}

\begin{abstract}
Keywords

- kinematics

- feline

- antebrachiocarpal joint

- carpus

- pronation/supination

Objective Cats rely on their forelimb mobility for everyday activities including climbing and grooming. Supination and pronation of the forelimb in cats are considered to primarily involve the antebrachium, rather than the carpus. Therefore, our null hypothesis was that there would be no movement of the carpal bones (radial carpal bone, ulnar carpal bone and accessory carpal bone) relative to the ulna during supination and pronation.

Study Design Eight feline cadaveric forelimbs were rotated from supination to pronation in a jig and computed tomography was performed in the neutral, supinated and pronated positions. The individual carpal bones were segmented from computed tomography images of the supinated and pronated scans in each of the eight specimens. A feline ulna coordinate system was established and used to quantify the translations and rotations between bones of the proximal carpal row and antebrachium.

Results After the carpus was rotated from the initial supinated position into pronation, there was significant translation ( $x, y$ and $z$ axes) and rotation ( $x$ and $y$ axes) of the proximal row of carpal bones based on absolute magnitude values. Given the differences in translations and rotations of the proximal row of carpal bones, our null hypothesis was rejected.

Conclusion The proximal row of carpal bones translate and rotate independently from the ulna in the cat during pronation of the antebrachium. This may have future implications in the diagnosis and management of feline carpal injuries involving the antebrachiocarpal joint.
\end{abstract}

\section{Introduction}

The carpus is a complex, multi-compartmental joint in which most of the uniaxial mobility of flexion and extension occurs at the larger, more proximal, antebrachiocarpal joint which is comprised of the distal end of the radius and ulna, and the radial carpal bone $(R C B)$, ulnar carpal bone (UCB) and accessory carpal bone (ACB). The middle carpal and carpometacarpal joints are more rigid and constrained by the short inter-carpal and carpometacarpal ligaments. ${ }^{1}$

received

January 16, 2019

accepted after revision

August 11, 2020

published online

November 20, 2020

It is supposition that the carpus of quadrupedal mammals, such as the dog, functions as a ginglymus, primarily allowing uniaxial motions of flexion and extension. ${ }^{2}$ Further, there are limited reports of normal carpal joint motion in cats. ${ }^{3,4}$ It is known that the antebrachium of the cat has a greater range of motion from pronation through to supination (115 degrees) than the $\operatorname{dog}$ (50 degrees), and this seems to be important for normal activities of cats, such as jumping, climbing and grooming. ${ }^{5}$

Cats commonly sustain injuries to the antebrachiocarpal joint in association with falling from a height or motor

(c) 2020. Thieme. All rights reserved. Georg Thieme Verlag KG,

Rüdigerstraße 14,

70469 Stuttgart, Germany
DOI https://doi.org/ 10.1055/s-0040-1719063. ISSN 0932-0814. 
vehicle accidents. Due to the traumatic nature of these injuries, ligamentous tearing is often associated with carpal bone fracture or luxation. ${ }^{6}$ Pancarpal arthrodesis therefore appears to be the most commonly employed surgical technique to treat these injuries. However, one study of the longterm outcome of pancarpal arthrodesis found approximately half of the cats had reduced ability to jump and climb. ${ }^{4}$ This was speculated to be due to restricted supination and pronation, which would suggest that mobility of the carpus is important in these joint movements. ${ }^{4}$

The kinematics of the individual carpal bones in the human wrist have been quantified using rigid body motion mathematics and analyses, which describe the motion of objects in space. ${ }^{7}$ The advantage of this methodology is that this allows the study of the motion of complex joints comprised of small bones and rigid body motion calculations in a non-invasive manner. In the present study, threedimensional imaging techniques were used to directly quantify the degree of movement of the proximal carpal bones and radius relative to the ulna in cadaveric feline specimens.

The aim of this study was therefore to describe normal kinematics of the feline antebrachiocarpal joint from supination to pronation and to determine whether the proximal row of carpal bones (RCB, UCB and $A C B$ ) translate and rotate independently of the ulna during these movements. Our null hypothesis was that there would be no movement of the carpal bones relative to the ulna from supination to pronation, which would indicate that the carpus translates and rotates as a single unit with the radius and ulna during rotation of the antebrachium.

\section{Materials and Methods}

\section{Description of Experimental Equipment}

A custom-made jig was used for testing each limb in supination and pronation (-Fig. 1). The construct was made from wooden blocks (dimension $4 \times 30 \times 15 \mathrm{~cm}$ ) arranged to create a platform that allowed for a carbon rod to suspend each antebrachium at the top of the jig.

The top of the jig consisted of two parallel blocks on either side separated by a $40 \mathrm{~mm}$ gap, which served as a platform for placement of the limb. The parallel blocks were separated by a $3.2 \mathrm{~mm}$ gap that allowed for sliding insertion of a carbon rod that suspended and fixed the proximal aspect of the antebrachium.

The base of the jig contained a $13 \mathrm{~mm}$ diameter hole that allowed for insertion of a $125 \mathrm{~mm}$ extension $12.7 \mathrm{~mm}$ diameter drive that was attached to a $6.4 \mathrm{~mm}$ female $\times 9.5 \mathrm{~mm}$ male socket adaptor with a universal joint (SidChrome, Sydney, Australia). This allowed for the attachment of a $32 \mathrm{~mm} 12$-point stainless steel socket. The base of the socket was covered in $5 \mathrm{~mm}$ high creative modelling compound (Kmart, Sydney, Australia) that prevented leakage of acrylic through the base of the socket. The limb was potted at the level of the mid-shaft of the metacarpals, as described below under 'Specimen preparation and mounting'. A $9.5 \mathrm{~mm}$ female $\times 12.7 \mathrm{~mm}$ male adaptor (Irwin, Sydney, Australia) was inserted at the lower end of the extension drive, which allowed attachment of a torque-measuring screw- driver (Torque Leader TT250 FH) during computed tomography (CT) scans. All components of the jig except for the socket were radiolucent to eliminate artifact during CT.

Two transverse wooden dowels were inserted through $4 \mathrm{~mm}$ diameter holes oriented perpendicular to the base of the jig (-Fig. 1). The limb was secured in supinated and pronated positions after applying a 250 Newton millimetre (N.mm) moment in each direction during image acquisition by using a quick grip clamp (Irwin, Sydney, Australia) at the base of the jig that was used to prevent movement of the extension drive by forcing the dowels centrally within the hole.

\section{Specimens}

Eighteen thoracic limbs were obtained from nine domestic short-haired cats weighing 2.9 to $4.9 \mathrm{~kg}$ that were euthanatized for reasons unrelated to the study and acquired in accordance with guideline GL001 from the University of Sydney animal ethics committee. The limbs were amputated at the level of the mid humerus and each limb was radiographed with orthogonal views of the carpus and elbow to ensure that they were free of skeletal disease. Cats were excluded from the study if they were less than 266 days old, as determined by the olecranon physis of the ulna being open. ${ }^{8}$ This was the case in one set of limbs, which was excluded from the study. There is currently no data to suggest that movement between left and right limbs of cats is different; therefore, we used a random number generator to determine which sided limb was used from each specimen, with one limb being selected from each cat. The remaining eight contralateral limbs were stored for another study. Each thoracic limb was wrapped in surgical gauze soaked with saline solution stored at $-20^{\circ} \mathrm{C}$.

\section{Specimen Preparation and Mounting}

Each limb was kept at room temperature for 12 hours prior to testing and soft tissues distal to the elbow were left intact. The digits were amputated at the level of the metacarpophalangeal joints. A $3.1 \mathrm{~mm}$ drill bit was used to create a transverse hole $\sim 5 \mathrm{~mm}$ proximal to the medial and lateral humeral epicondyles and a $3.2 \mathrm{~mm}$ carbon rod was inserted in a medial to lateral direction. A drill guide was used to ensure that there was a consistent trajectory between specimens. The limb was suspended in the testing jig and the manus (at the level of the distal metacarpals III and IV) was potted in acrylic within the previously described socket. The acrylic was allowed to polymerize and harden for 30 minutes prior to CT. Alignment between limbs in the jig was standardized by ensuring that the olecranon tuber was orientated perpendicular to the base of the jig, and the angle of the carpus was 180 degrees. The soft tissues of the elbow were left intact and there was unconstrained movement of the elbow.

\section{Specimen Testing and CT Acquisition}

The jig containing the cadaveric limb was positioned in a CT scanner (16 slice Philips Brilliance helical CT, Amsterdam, Netherlands) and imaged using the following parameters: $120 \mathrm{KV}, 200 \mathrm{mAs}$, slice thickness $0.8 \mathrm{~mm}$ and ultra-high resolution. Each limb was imaged from the distal humerus 


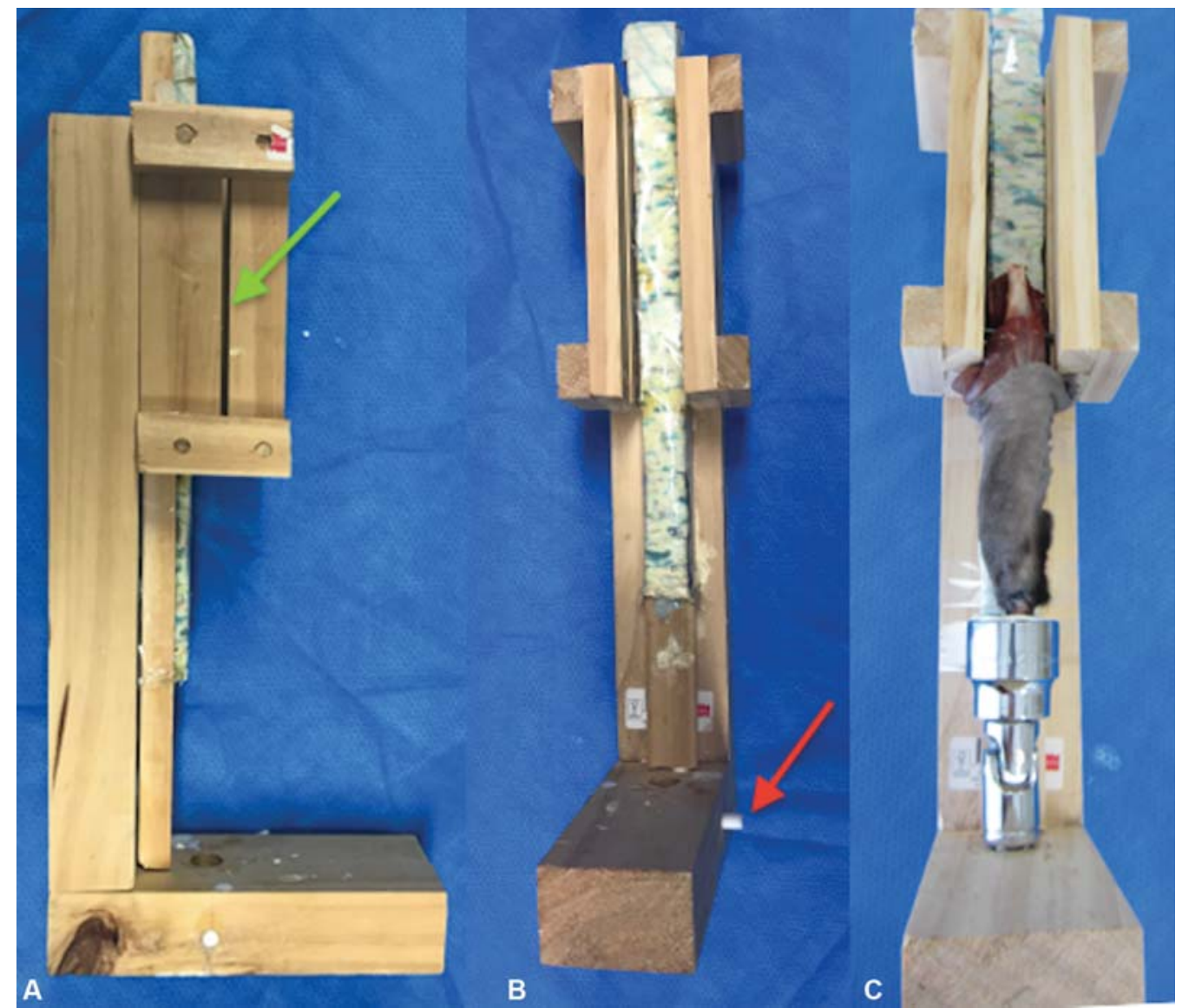

Fig. 1 Part A shows side on view of testing jig, B shows frontal view of testing jig without a mounted specimen and C shows frontal view of testing jig with a mounted specimen. The jig was constructed from wooden blocks. The specimen was suspended from a carbon rod with sliding insertion between parallel blocks to allow for adjustment of the limb position according to limb length. The wooden dowels in the base of the jig were secured centrally to fix the extension drive in a pronated or supinated position using a clamp. The green arrow points to the gap between parallel blocks that allowed for sliding insertion of a carbon rod, with the height adjusted to limb length. The red arrow highlights wooden dowels that are secured centrally within holes to fix the extension drive in a pronated or supinated position between computed tomography scans.

to the metacarpals in a neutral position and then with a 250 N.mm supinating moment, and then with a 250 N.mm pronating moment ( - Video 1 ). There were three sets of scans for each antebrachium tested (a total of 24 scans). Moment (N.mm) was applied using the torque-measuring screwdriver attached to a universal joint. Computed tomography data were stored in a digital imaging and communication in medicine (DICOM) format for later processing.

\section{Video 1}

Movie demonstrating all components of the testing jig. The forelimb is suspended by a carbon rod inserted transversely proximal to the humeral epicondyles, and the distal manus is potted in acrylic. The limb is pronated and supinated using a torque screwdriver attached to a universal joint and extension drive. Online content including video sequences viewable at: https://www.thieme-connect.com/products/ ejournals/html/10.1055/s-0040-1719063.

\section{Image Segmentation}

The DICOM images were imported into a DICOM image processing software (Materialise Mimics version 19.0, Leuven Belgium) that produces three-dimensional (3D) models, and thresholded as previously described. ${ }^{9}$ The following bones were segmented: radius, ulna, RCB, UCB and $\mathrm{ACB}$ and $3 \mathrm{D}$ iso-surface models were

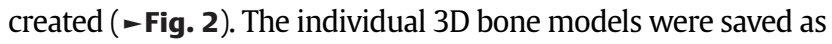
surface tessellation language/stereolithography file types.

\section{Alignment of Specimens within the Global Coordinate System}

This definition of the feline carpus coordinate system was a modified version of a human wrist definition. ${ }^{10}$ A right ulna, $3 \mathrm{D}$ reconstructed from the $\mathrm{CT}$ of a supinated specimen from the present study, was used to define the feline ulna global coordinate system (GCS) (-Fig. 3; - Appendix Fig. 1, available in the online version).

The feline ulna coordinate system definition was the same as the human ulna coordinate system in that the x-axis was defined as the long axis of the ulna (defined as the principal axis via a singular value decomposition of the vertices making up the ulna model surface) and the coordinate system origin $(\mathrm{x}, \mathrm{y}, \mathrm{z} 0,0,0)$ was placed at the centre point 


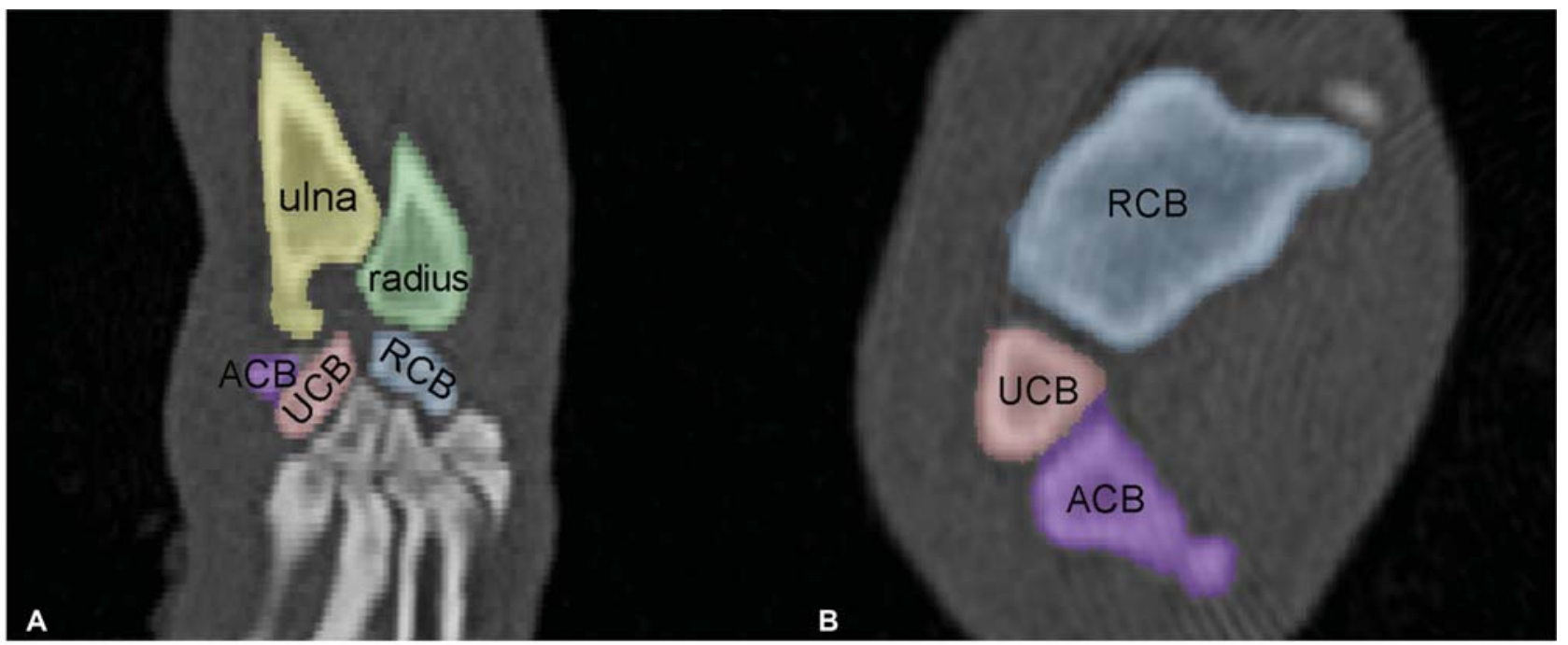

C

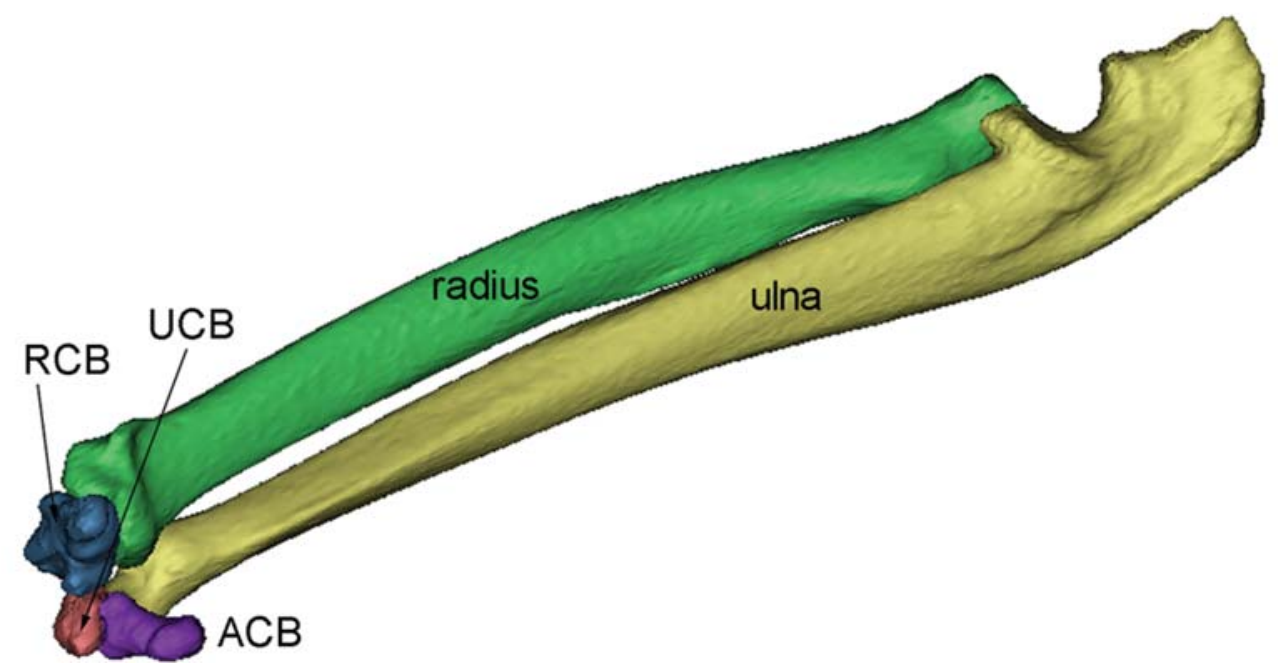

Fig. 2 Steps in the segmentation process. (A) Segmentation of the individual bones (right hand side bones, ulna in yellow; radius in green, radial carpal bone $[R C B]$ in blue, ulnar carpal bone $[\mathrm{UCB}]$ in red and accessory carpal bone $[A C B]$ in purple) from a dorsal slice of the computed tomography (CT) Digital Imaging and Communication in Medicine (DICOM) image stack. The radius is to the right of the ulna, the ACB is distal to the ulna and the RCB is distal to the radius in this image. (B) Close up of a single transverse slice from the CT DICOM image stack with ACB, UCB, RCB segmented. (C) Three-dimensional iso-surface models of carpal bones (colour scheme as in A and B).

of the distal ulna articular surface. ${ }^{10}$ The centre point of the ulna distal articular surface was defined by projecting (using the $\mathrm{x}$-axis vector) the centroid (mean) point of the distal articular surface vertices until this centroid point intersected the distal ulna articular surface. The ulna was then translated so that this intersection point was at $0,0,0$.

The feline carpal coordinate system differed from the human coordinate system in that in the present definition a pre-zaxis was defined as the dorsal-palmar depth of the ulna at the level of the proximal ulnar joint (-Fig. 3).$^{10}$ The y-axis in the present study was defined as the cross-product of the $x$ - and pre-z-axes, with the $z$-axis being defined as the cross-product of the $x$ - and $y$-axes. Taking the cross-products of the axes in this way ensures that all three axes are orthogonal ( - Fig. 3 ).

The aligned ulna was named the 'reference' bone (a right ulna from a supinated scan). In the ulna coordinate system as defined in the present study, rotation around the $\mathrm{x}$-axis corresponded to pronation/supination, rotation around the $y$-axis refers to flexion/extension and rotation around the zaxis refers to varus/ valgus (-Fig. $\mathbf{3}$ ).

\section{Relative Bone Position and Calculation of Kinematics} Software (Materialise 3Matic, version 11.0, Leuven, Belgium) was used to orient the bones from each specimen in both supinated and pronated configurations within the GCS. This was achieved by iterative closest point superimposition of the specimen bones to the scaled reference ulna. ${ }^{11}$ In the case where the specimen was a left limb, the specimen ulna was mirrored in the $\mathrm{xz}$ plane (normal $=\{0,1,0\}$ ) so that all ulnas were right sided.

During this process, the specimens were not scaled, but instead a copy of the reference bone was made and scaled to the length of the ulna of each of the eight specimens. The reference ulna remained static and was not moved within 


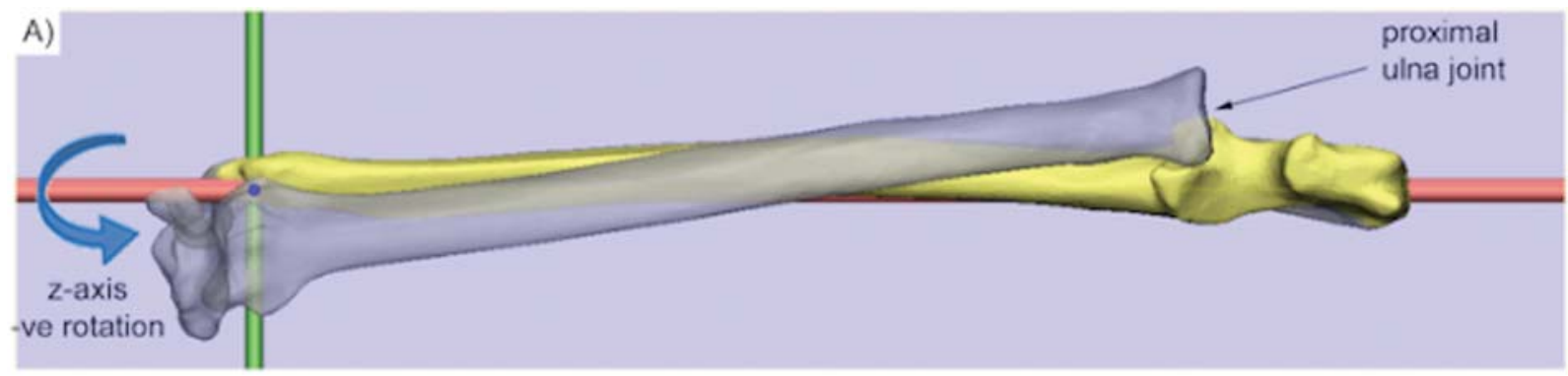

B)

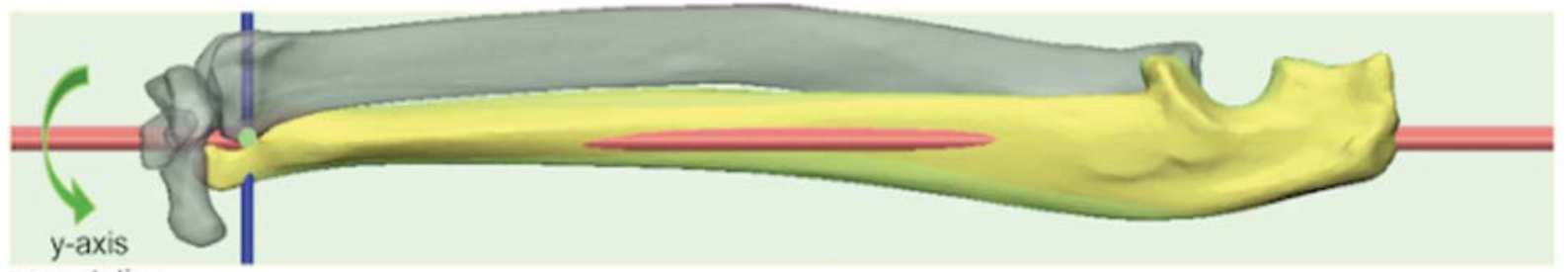

+ ve rotation

C)

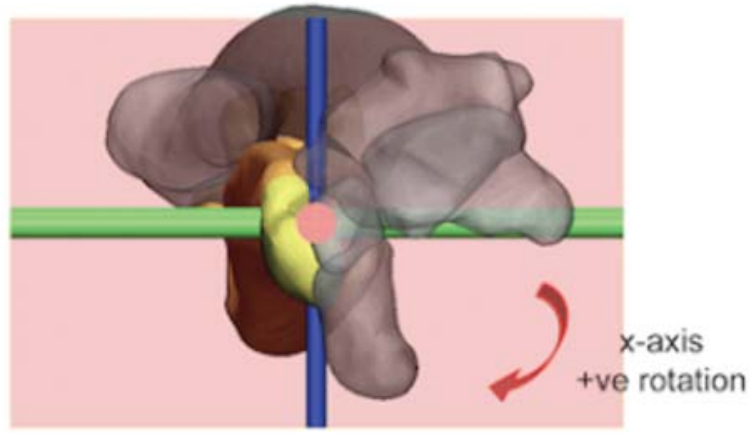

D)

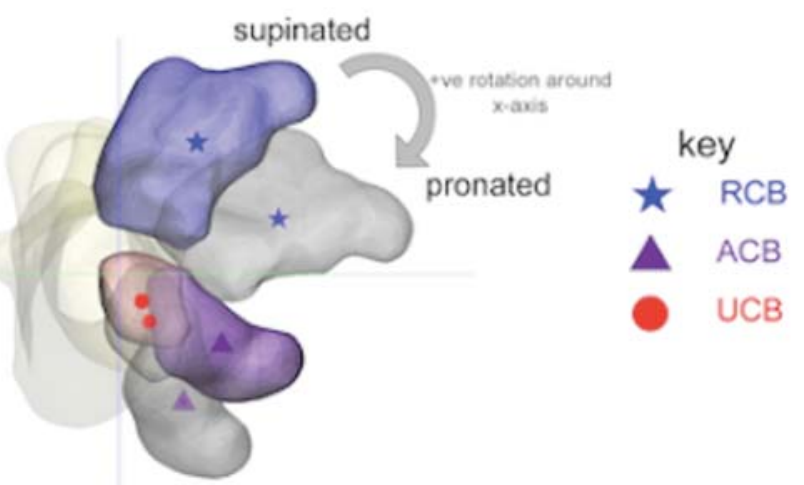

Fig. 3 Ulna coordinate system (right ulna shown). The antebrachium is viewed in all three planes as defined by the right ulna coordinate system. The bones are viewed in pronation in parts A, B, C. Panel A corresponds to a dorsopalmar view; B is medial to lateral, while C and D are both distal to proximal and show how the ulna is aligned with the z-axis using the proximal ulna anatomy. The bones are coloured in supination and grey in pronation. Pronation/supination rotations predominantly occur around the $x$-axis (red cylinder) with pronation being positive rotation as shown by the red arrow in $\mathbf{C}$ and the grey arrow in $\mathbf{D}$. The y-axis (green cylinder) represents flexion/extension with flexion being positive (B) and the z-axis (blue cylinder) represents radial/ulnar deviation (varus/valgus) with ulnar deviation (valgus) being positive (A). Note that a consequence of using the ulna coordinate system is that when the limb is moved from supinated to pronated positions, the alignment of the carpal bones with the $y$ and $z$ axes effectively changes meaning that flexion-extension will occur around the $y$-axis in pronation, but more around the z-axis in supination. We have defined the movements based on a pronated antebrachium. Stars, triangles and circles in D indicate centroids of the bone models (translucent points indicate centroids of grey bone models, opaque points indicate centroids of coloured bones). In this rotation (coloured grey), the radial carpal bone has undergone a negative translation along the $y$-axis, while the accessory carpal bone has undergone a positive translation along the $y$-axis.

studies to ensure that relative bone position was accurate. The specimen bones were then aligned within the GCS by superimposition of the specimen ulna with the scaled reference ulna. Length measurements were taken from the centre of the distal articular facet surface at the position, where $\{0,0,0\}$ is located in the reference ulna, to the proximal most point of the olecranon process. During the alignment process of the specimen ulna to the reference ulna, the other bones (radius, RCB, UCB and $A C B$ ) of the specimen were moved (translated and rotated) with the specimen ulna. ${ }^{9}$ This resulted in the specimen bones maintaining their relative configuration (supinated or pronated 
configuration), while being meaningfully oriented within the GCS. A set of axes was not defined for each specimen because this method has been shown to be less accurate than aligning specimens with a reference bone using iterative closest point superimposition. ${ }^{12}$

\section{Calculation of Translations and Rotations}

The new position of the 3D surface models of the bones of interest was saved and this allowed a translation vector (millimetres moved in $\mathrm{x}, \mathrm{y}$ and $\mathrm{z}$ ) and rotation matrix (a $3 \times 3$ matrix) for each bone movement from supination to pronation position to be calculated. The translation vector was calculated as the difference between the centroids (mean of all 3D vertices in the mesh) of two 3D surface model positions (in millimetres) (-Fig. 3). The rotation matrix was calculated using a singular value decomposition algorithm implemented in a software programme used in mathematical applications (Mathematica, version 10.1, Wolfram, Illinois, United States). The rotation matrix rotated the 3D surface model from supinated to pronated orientation. The rotation matrix was then decomposed into the rotational components occurring around the $\mathrm{x}, \mathrm{y}$ and $\mathrm{z}$ axes, and this was expressed as degrees of movement. ${ }^{9}$

Values were calculated for the radius, RCB, UCB and $A C B$ relative to the ulna by determining the $\mathrm{x}, \mathrm{y}$ and $\mathrm{z}$ values in translations (millimetres) and rotations (degrees) for the eight specimens used in the study. Rotations were calculated with respect to the ulna coordinate system ( - Fig. 2). The means of the results of the eight limbs were calculated for the translations and rotations of each bone (radius, RCB, UCB, ACB) aligned to the ulna. Total range of motion values for bone rotations of each specimen from supination to pronation were also defined by calculating the degree of rotation around a single helical axis using previously described methods. ${ }^{13,14}$

\section{Statistical Analysis}

Statistical analyses were performed using commercially available statistical packages ([Genstat] VSNi Hemel Hempstead UK; and Mathematica, version 10, Champaign, Illinois, United States).

A linear mixed model was utilized to investigate the association between bone type and absolute magnitude of translation and rotation, with a random term of Animal. Directionality of both translation and rotation of the bones (positive or negative) was removed from the raw values prior to performing these calculations to indicate absolute size of change relative to zero. Absolute rather than raw values were used to test the null hypothesis that there was no movement of the proximal carpal bones and radius relative to the ulna. Anderson-Darling tests were conducted to ensure the underlying assumptions of normality were met. Post-hoc least significant difference analyses were performed to determine pairwise differences. For all statistical analyses, a $p$-value of $<0.05$ was considered significant.

A general linear regression was then used to determine the degree of coupling between individual bones based on the results for bone rotations. The ulna was excluded because this was the static reference bone to which the motion of the other bones was related. Results were presented for combinations of all bones (RCB, UCB, radius, $A C B$ ), in the following pairs: the RCB and $U C B$; RCB and radius; $U C B$ and $A C B ; R C B$ and $A C B$; radius and $A C B$; the radius and UCB. Values approaching one indicate high correlation in the total range of motion of the two bones.

\section{Results}

\section{Translations}

The mean translation for each of the bones varied depending on their distance from the distal ulna articular surface, which

Table 1 Magnitude and direction of translation and rotation of each bone (mean \pm standard deviation, maximum and minimum) from supination to pronation $(n=8)$

\begin{tabular}{|c|c|c|c|c|c|c|c|}
\hline Bone & Axis $^{a}$ & $\begin{array}{l}\text { Mean distance } \\
\pm \mathrm{SD}(\mathrm{mm})\end{array}$ & $\begin{array}{l}\text { Max distance } \\
(\mathrm{mm})\end{array}$ & $\begin{array}{l}\text { Min distance } \\
(\mathrm{mm})\end{array}$ & $\begin{array}{l}\text { Mean angle } \\
\pm \text { SD (degrees) }\end{array}$ & $\begin{array}{l}\text { Max angle } \\
\text { (degrees) }\end{array}$ & $\begin{array}{l}\text { Min angle } \\
\text { (degrees) }\end{array}$ \\
\hline \multirow{3}{*}{$\begin{array}{l}\text { Accessory } \\
\text { carpal bone }\end{array}$} & $x$ & $-0.17 \pm 1.34$ & 1.51 & -2.10 & $41.94 \pm 9.33$ & 55.33 & 25.76 \\
\hline & $y$ & $2.02 \pm 0.60$ & 3.39 & 1.59 & $0.13 \pm 9.96$ & 13.68 & -10.56 \\
\hline & $z$ & $-0.78 \pm 2.06$ & 2.10 & -3.50 & $-6.47 \pm 7.62$ & 6.51 & -16.04 \\
\hline \multirow[t]{3}{*}{ Radius } & $x$ & $0.13 \pm 0.57$ & 0.72 & -0.80 & $30.27 \pm 10.78$ & 49.59 & 9.99 \\
\hline & $y$ & $-2.46 \pm 0.81$ & -1.04 & -3.95 & $0.14 \pm 1.75$ & 2.87 & -1.53 \\
\hline & $z$ & $-0.30 \pm 1.10$ & 1.05 & -1.90 & $-2.60 \pm 1.08$ & -0.94 & -4.67 \\
\hline \multirow{3}{*}{$\begin{array}{l}\text { Radial } \\
\text { carpal bone }\end{array}$} & $x$ & $0.10 \pm 0.80$ & 1.22 & -1.21 & $45.11 \pm 12.02$ & 61.05 & 19.41 \\
\hline & $y$ & $-3.93 \pm 1.40$ & -1.53 & -6.55 & $-0.25 \pm 4.10$ & 5.30 & -6.91 \\
\hline & z & $-1.02 \pm 3.26$ & 3.43 & -4.49 & $-6.36 \pm 6.76$ & 2.02 & -17.29 \\
\hline \multirow{3}{*}{$\begin{array}{l}\text { Ulnar } \\
\text { carpal bone }\end{array}$} & $x$ & $0.05 \pm 0.48$ & 0.73 & -0.73 & $44.97 \pm 14.21$ & 60.30 & 17.81 \\
\hline & $y$ & $-0.63 \pm 0.53$ & 0.31 & -1.51 & $0.99 \pm 6.14$ & 10.37 & -7.29 \\
\hline & $z$ & $0.03 \pm 0.82$ & 1.07 & -1.08 & $-8.60 \pm 6.16$ & -2.25 & -21.12 \\
\hline
\end{tabular}

Abbreviation: SD, standard deviation.

${ }^{a}$ Rotation occurs around the axis (degrees), translation occurs along the axis (millimetres). Positive and negative values denote the direction of rotation and translation; positive in the $y$-axis refers to pronation, positive in the $y$-axis is flexion and in the positive in the $z$-axis is valgus. The negative values refer to the inverse direction. Values for each specimen are based on scaling to a reference ulna. 
Table 2 Absolute magnitude of translation and rotation of each bone (mean \pm standard error) from supination to pronation $(n=8)$. Translations are in millimetres, and rotations are degrees

\begin{tabular}{|l|l|l|l|l|l|l|l|}
\hline & Axis & ACB & Radius & RCB & UCB & Standard error & $p$-Value \\
\hline \multirow{5}{*}{ Translation (mm) } & $\mathrm{x}$ & $1.1887^{\mathrm{a}}$ & $0.5063^{\mathrm{b}}$ & $0.68^{\mathrm{b}}$ & $0.3788^{\mathrm{b}}$ & 0.1204 & $<0.001$ \\
\cline { 2 - 8 } & $\mathrm{y}$ & $2.023^{\mathrm{a}}$ & $2.461^{\mathrm{a}}$ & $3.926^{\mathrm{b}}$ & $0.709^{\mathrm{c}}$ & 0.0313 & $<0.001$ \\
\cline { 2 - 8 } & $\mathrm{z}$ & $1.945^{\mathrm{a}}$ & $0.936^{\mathrm{b}}$ & $3.038^{\mathrm{c}}$ & $0.654^{\mathrm{b}}$ & 0.271 & $<0.001$ \\
\hline \multirow{3}{*}{ Rotation (degrees) } & $\mathrm{x}$ & $41.94^{\mathrm{a}}$ & $30.27^{\mathrm{b}}$ & $45.11^{\mathrm{a}}$ & $44.97^{\mathrm{a}}$ & 4.14 & $<0.001$ \\
\cline { 2 - 8 } & $\mathrm{y}$ & $8.451^{\mathrm{a}}$ & $1.449^{\mathrm{b}}$ & $3.315^{\mathrm{b}, \mathrm{c}}$ & $5.08^{\mathrm{c}}$ & 1.001 & $<0.001$ \\
\cline { 2 - 8 } & $\mathrm{z}$ & 8.099 & 2.599 & 6.868 & 8.599 & 1.838 & 0.093 \\
\hline
\end{tabular}

Abbreviations: ACB, accessory carpal bone; RCB, radial carpal bone; UCB, ulnar carpal bone.

$a, b, c$ Mean values with different superscript letters within a row are significantly different from each other.

is the origin of the ulna coordinate system (GCS x, y, z 0, 0, 0, from which all motion was measured) (-Appendix Table 1,

- Appendix Graph 1, available in the online version).

Based on the absolute magnitude values, the ACB had a significantly greater absolute magnitude of translation along the $\mathrm{x}$-axis compared with all other bones $(p<0.001$; - Table 2). The RCB had significantly higher absolute magnitude of translation values for bothy-and z-axes compared with all other bones ( $p<0.001$, - Table 2$)$.

\section{Rotations}

\section{Direction of Rotation}

The rotations were greatest around the $x$-axis (in the yz plane), followed by the $\mathrm{z}$ - and y-axes ( - Table $\mathbf{1}$; - Appendix Table 2 , available in the online version; - Appendix Graph 1, available in the online version). The values for movements around the $\mathrm{x}$-axis were positive as the bones moved from a supinated configuration to a pronated configuration (-Table 1; -Fig. 3). Rotations around the y-axis were varied (positive and negative), indicating that flexion and/or extension rotations did not form part of the supination to pronation movement as simulated here. The standard deviation for rotation of each of the carpal bones was largest in the $\mathrm{x}$-axis.

Table 3 Rank of carpal bone kinematic coupling based on $R^{2}$ value from regression analyses of total range of rotation of paired bones after change in position from supination to pronation (-Appendix Table 3, available in the online version)

\begin{tabular}{|l|l|l|l|}
\hline Rank & Bone on x-axis & Bone on y-axis & $R^{2}$ \\
\hline 1 & RCB & UCB & 0.9203 \\
\hline 2 & RCB & Radius & 0.9023 \\
\hline 3 & UCB & ACB & 0.8363 \\
\hline 4 & RCB & ACB & 0.8319 \\
\hline 5 & Radius & ACB & 0.7772 \\
\hline 6 & Radius & UCB & 0.7488 \\
\hline
\end{tabular}

Abbreviations: $A C B$, accessory carpal bone; $R C B$, radial carpal bone; UCB, ulnar carpal bone.

\section{Magnitude of Rotation}

There was minimal movement of all bones around the y-axis (flexion-extension), compared with rotations around the x-axis (supination-pronation), with slight rotation contributions around the z-axis (varus/valgus). In specimen 3, there was half the amount of rotation compared with the other specimens in the study.

The absolute magnitude rotations in the z-axis for the RCB (6.868 degrees), UCB (8.599 degrees) and ACB (8.099 degrees) were not significantly different to the radius (2.599 degrees) $(p=0.093)$. The rotation between the radius and ulna (mean 30.3 degrees) was significantly less than between the ulna and all other bones $(p<0.001,-$ Table 2$)$.

\section{Linear Regression Analysis}

Pairwise comparisons were made to determine the degree of coupling between total ranges of motion of individual bones based on rotation results from a supinated to pronated position (-Appendix Table 3, available in the online version; - Table 3).

\section{Discussion}

Our null hypothesis, that there would be no movement of the carpal bones relative to the ulna during supination and pronation, was rejected on the basis of both absolute translation and rotation results (-Table 2 ). With the exception of rotation in the z-axis, rotations and translations of all bones in the three axes were significantly greater than zero (-Table 2). Rejection of the null hypothesis therefore indicates that the feline carpal joint is not purely a ginglymus because movements other than flexion and extension at the level of the antebrachiocarpal joint may occur during forelimb motion. Although beyond the scope of the present study, this may have implications in the future management of feline carpal injuries, particularly when there is involvement of the antebrachiocarpal joint.

The results for supination and pronation were relatively low (greatest value 61 degrees) compared with the aforementioned normal ranges of supination and pronation in cats because moment, rather than total range of motion, was used to standardize measurements between limbs. This indicates that natural movements of the feline forelimb between supination and pronation require a moment greater than 250 N.mm (whether this moment is active or passive) 
(-Appendix Table 3, available in the online version, for total range of motion). This may also account for the variation in standard deviation for each of the carpal bones in the $\mathrm{x}$-axis.

The present study demonstrates that similar to humans, the kinematics of the feline carpal bones are complex and do not occur in a single plane alone. Flexion and extension are the primary plane of movement that is usually reported, although varus/valgus and supination/pronation have been alluded to in a previous study of cats. ${ }^{15}$ The ACB rotated to a lesser degree than the remaining proximal carpal row. This finding is, perhaps, unsurprising because the articulation of the ACB with the carpus is small. ${ }^{16,17}$ The ACB also consistently had positive values (flexion) in the y-axis, with the remaining bones translating in the opposite direction, which is possibly because of the resistance to rotation from the insertion for the flexor carpi ulnaris muscle.

Computed tomography was selected for initial data capture over more invasive methods for tracking moment because it is a non-invasive technique that does not require dissection of the carpus and potential damage to important soft tissue anatomical structures such as the dorsal joint capsule. $^{7}$ As the largest bone in the proximal carpal row (RCB) is $\sim 6 \mathrm{~mm}$ diameter, it would also be technically difficult to insert a marker without causing iatrogenic fracture or displacement. ${ }^{18}$ This has been shown to be an accurate method for measuring changes in position of small bones with variations in resolution, segmentation threshold and reconstruction smoothing algorithms having minimal effect on the accuracy (precision and trueness) of kinematic measurements (e.g. translation imprecision was $0.5 \mathrm{~mm}$, which is less than the slice thickness of the CT scanner used in the present study $[0.8 \mathrm{~mm}])^{9}{ }^{9}$

Inconsistency in the direction of rotation for the same bone between specimens may be due to natural variation between specimens and individual bone movements. ${ }^{2,19}$ This is similar to the in vivo findings of the tarsal bones in humans. ${ }^{19}$ Due to this being a cadaveric study, the effect of weight bearing and active stabilizers such as the pronator and supinator muscles on this supination/pronation was not present. This potentially could have affected the interaction and position of individual carpal bones.

Apparent inconsistency in movements, particularly translations, between bones in the joint complex of an individual specimen may further be a consequence of the coordinate system definition. For example, if the coordinate system is defined as passing between the ACB and RCB as in our study ( - Fig. 3), then rotation from supination to pronation will result in a larger positive displacement of the RCB, and large negative displacement of the UCB. Note that this does not mean that the carpal bones are moving separately or disassociating but is a consequence of the standard coordinate system definition.

The RCB rotated in an opposite direction to the other bones around the y-axis. This y-axis rotation was also relatively minor as the predominant rotation was around the $\mathrm{x}$-axis for all bones. The reason for the RCB rotation being in the opposite direction around the $y$-axis to the other bones (radius, $\mathrm{UCB}, \mathrm{ACB}$ ) is that the axis of rotation of the RCB was aligned differently from the other bones. Whereas the axes about which the radius, UCB and ACB rotated were similarly aligned (somewhat close to the ulna $x$-axis), the axis about which the RCB rotated differed. The axis about which the RCB rotated differed by having a mediolateral component, rather than being predominantly proximal-distal in orientation (close to the ulna $\mathrm{x}$-axis) as with the other bone rotation axes.

There was some variation in the magnitude of results reported in this study. In particular, specimen 3 had half the amount of rotation compared with other specimens, which may have been due to slipping of the digits within the potted acrylic after application of the rotational moment. In specimen 8, there was the greatest translation of the RCB in the yaxis $(6.55 \mathrm{~mm})$. These values are greater than expected but may be explained by rotational force being applied to the distal limb, thereby opening the joint spaces and causing greater stretching of the ligaments.

In cats, it has been demonstrated that there is ulnar deviation (valgus) of the carpus and supination of the forelimb with ulnar deviation (valgus) of the manus during the swing phase. ${ }^{3}$ Although the present study is cadaveric with load control used to apply torque to the limbs, we similarly showed that supination of the forelimb was coupled with ulnar deviation (valgus) of the carpus and thereby manus (-Table 1; - Fig. 3 ).

As all of the bones tested are part of an anatomical unit (the carpus) and none of the bones disassociated or dislocated during the movements between supination and pronation, relatively high correlation in the bone total range of motion during rotation was to be expected (-Appendix Table 3, available in the online version; - Table 3). Translation results were considered to be less important because they vary depending on proximity of the individual bones to the ulnar coordinate system origin.

Cats and dogs have an inter-carpal ligament that connects the RCB and UCB. ${ }^{1,20}$ Based on the linear regression analysis, the results of the present study showed a high degree of coupling in the total range of motion for the RCB and UCB $\left(R^{2}=0.920\right)$, suggesting that the RCB and UCB rotate as a functional unit (-Table 3). This close relationship between the RCB and UCB has not previously been described in the cat, but presumably the inter-carpal ligament between this pair of bones must be disrupted when traumatic RCB luxation occurs. ${ }^{20}$

There are limitations of our study. It may have been preferable to supinate/pronate the carpus by fixing the elbow and rotating the distal limb from the level of the elbow; however, this is difficult to standardize in an ex vivo study. The carpus was also only tested with 180 degrees of extension, and the results may have varied if the carpus was tested in different positions (such as flexion, extension and under load). ${ }^{21}$ Only metacarpals III and IV were potted in acrylic, which also may have produced different results to fixing the entire manus.

Due to the raw data having positive and negative values, the mean values were close to zero, although our statistical analysis did not take into account the direction of movement.

The intent of our study was to provide a foundation on which our knowledge of feline carpal kinematics can be based for future studies on feline pancarpal arthrodesis, 
because it is important to understand the extent to which this surgical procedure may compromise supination and pronation. As stated in the introduction, the assumption that pancarpal arthrodesis restricts supination and pronation has only been reported anecdotally. ${ }^{4,22}$

\section{Conclusion}

In this model in which there was unconstrained movement of the elbow and carpus, it was concluded that the feline carpus (and specifically, the bones of the proximal carpal row) rotates and translates independently of the ulna during supination to pronation movement of the antebrachium.

\section{Note}

This research was presented at the 27th European College of Veterinary Surgeons annual scientific meeting on July 6 , 2018 in Athens, Greece.

\section{Authors' Contributions}

All authors contributed to conception of study, study design, acquisition of data, data analysis and interpretation, and drafting/revising and approving the submitted manuscript. They are publically accountable for relevant content.

\section{Funding}

R.B. was funded by a residency stipend from the Sydney School of Veterinary Science, Faculty of Science. The use of image processing software was subsidized by the Surgical and Orthopaedic Research Laboratory, Prince of Wales Clinical School.

\section{Conflict of Interest}

None declared.

\section{Acknowledgments}

The authors would like to thank Dr Evelyn Hall for her contribution in assisting with statistical analysis of data, and Mrs Helen Laurendet for her contribution in CT image acquisition.

\section{References}

1 Yalden DW. The functional morphology of the carpal bones in carnivores. Acta Anat (Basel) 1970;77(04):481-500

2 Neville-Towle JD, Tan CJ, Parr WCH, Walsh WR, Johnson KA. Three-dimensional kinematics of the canine carpal bones imaged with computed tomography after ex vivo axial limb loading and palmar ligament transection. Vet Surg 2018;47(06):861-871
3 Caliebe F, Häubetaler J, Hoffmann P, Illert M, Schirrmacher J, Wiedemann E. Cat distal forelimb joints and locomotion: an Xray study. Eur J Neurosci 1991;3(01):18-31

4 Calvo I, Farrell M, Chase D, Aisa J, Rayward R, Carmichael S. Carpal arthrodesis in cats. Long-term functional outcome. Vet Comp Orthop Traumatol 2009;22(06):498-504

5 Roos H, Brugger S, Rauscher T. [The biological valence of the motion in the radioulnar joints of the cat and dog]. Anat Histol Embryol 1992;21(03):199-205

6 Basa RM, Johnson KA. Management of feline carpal injuries: what are the options and when is arthrodesis indicated? J Feline Med Surg 2019;21(09):809-823

7 Moojen TM, Snel JG, Ritt MJPF, Kauer JMG, Venema HW, Bos KE. Three-dimensional carpal kinematics in vivo. Clin Biomech (Bristol, Avon) 2002;17(07):506-514

8 Smith RN. Fusion of ossification centres in the cat. J Small Anim Pract 1969;10(09):523-530

9 Tan CJ, Parr WCH, Walsh WR, Makara M, Johnson KA. Influence of scan resolution, thresholding, and reconstruction algorithm on computed tomography based kinematic measurements. J Biomech Eng 2017;139(10):1-5

10 Coburn JC, Upal MA, Crisco JJ. Coordinate systems for the carpal bones of the wrist. J Biomech 2007;40(01):203-209

11 Besl PJ, McKay ND. Method for registration of 3-D shapes. Proc. SPIE 1611, Sensor Fusion IV: Control Paradigms and Data Structures. 1992:586-606

12 Parr WCH, Chatterjee HJ, Soligo C. Calculating the axes of rotation for the subtalar and talocrural joints using 3D bone reconstructions. J Biomech 2012;45(06):1103-1107

13 Spoor CW, Veldpaus FE. Rigid body motion calculated from spatial co-ordinates of markers. J Biomech 1980;13(04):391-393

14 Panjabi MM. Centers and angles of rotation of body joints: a study of errors and optimization. J Biomech 1979;12(12):911-920

15 Miller S, Van Der Meché FGA. Movements of the forelimbs of the cat during stepping on a treadmill. Brain Res 1975;91(02): 255-269

16 Mikić ZD, Ercegan G, Somer T, Somer T. Detailed anatomy of the antebrachiocarpal joint in dogs. Anat Rec 1992;233(02): 329-334

17 Guilliard MJ. Dorsal radiocarpal ligament sprain causing intermittent carpal lameness in high activity dogs. J Small Anim Pract 1997;38(10):463-465

18 Simpson D, Goldsmid S. Pancarpal arthrodesis in a cat: a case report and an anatomical study. Vet Comp Orthop Traumatol 1994;7(01):45-50

19 Lundgren P, Nester C, Liu A, et al. Invasive in vivo measurement of rear-, mid- and forefoot motion during walking. Gait Posture 2008;28(01):93-100

20 Pitcher GDC. Luxation of the radial carpal bone in a cat. J Small Anim Pract 1996;37(06):292-295

21 Bitton E, Joseph R, Portman L, et al. The effect of extension and loading of the carpus on radial rotation. Vet Surg 2013;42(08): 909-917

22 Voss K, Geyer H, Montavon PM. Antebrachiocarpal luxation in a cat: A case report and anatomical study of the medial collateral ligament. Vet Comp Orthop Traumatol 2003;16; (04):266-270 